\title{
Tissue at risk concept for endovascular treatment of severe vasospasm after aneurysmal subarachnoid haemorrhage
}

\author{
J Beck, A Raabe, H Lanfermann, V Seifert, S Weidauer
}

J Neurol Neurosurg Psychiatry 2004;75:1779-1781. doi: 10.1136/jnnp.2004.036921

\begin{abstract}
Objective: To report a case of severe vasospasm after subarachnoid haemorrhage (SAH) where "tissue at risk" was identified by magnetic resonance imaging (MRI), and to demonstrate the haemodynamic consequences with either resolution of the perfusion-diffusion mismatch by balloon angioplasty or evolution of an infarct.

Methods: A 45 year old women with SAH underwent surgical treatment of a ruptured middle cerebral artery (MCA) aneurysm. On day 3 she became obtunded and developed a right hemiparesis. Diffusion weighted (DWI) and perfusion weighted (PWI) imaging were done before and after transluminal balloon angioplasty (TBA) of multifocal proximal vasospasm.

Results: The initial MRI revealed no DWI lesion but PWI showed a severe perfusion deficit of 6.7 to 16.4 seconds in the complete left MCA territory. Digital subtraction angiography confirmed severe segmental narrowing of left $\mathrm{Cl}$ and M1. The spastic segments were successfully dilated by TBA. Follow up MRI showed that the PWI-DWI mismatch resolved in the anterior and middle MCA territory with no tissue infarction, whereas in the terminal dorsal MCA territory a severe mismatch remained and cerebral infarction evolved. Conclusions: PWI/DWI can identify tissue at risk for infarction in severe vasospasm following SAH. This may allow selection of patients for angioplasty and the monitoring of treatment effects.
\end{abstract}

D espite much effort and progress the early diagnosis of cerebral ischaemia from vasospasm after subarachnoid haemorrhage remains a serious obstacle in the treatment of patients with ruptured intracranial aneurysms. ${ }^{1}$ With a combination of perfusion weighted (PWI) and diffusion weighted (DWI) imaging it is possible to detect areas with a perfusion-diffusion mismatch-that is, areas of misery perfusion where the tissue is ischaemic but not yet infarcted. ${ }^{2}$ This "tissue at risk" concept derives from studies of ischaemic stroke, grossly identifies the ischaemic penumbra, and has proved to be a valuable tool for selecting patients for intravenous or intra-arterial thrombolysis. ${ }^{3}$

We hypothesise that the tissue at risk concept for cerebral infarction also applies to patients with vasospasm after subarachnoid haemorrhage and we present a case where a PWI-DWI mismatch was identified. Segmental arterial narrowing was thought to have been the cause for misery perfusion and was treated with transluminal balloon angioplasty (TBA). Follow up magnetic resonance imaging (MRI) revealed areas of resolution of the mismatch with no infarction and areas with remaining mismatch where an infarct developed. This case indicates that the tissue at risk concept may also apply to misery perfusion caused by vasospasm after subarachnoid haemorrhage.

\section{CASE REPORT}

A 45 year old women was admitted to the department of neurosurgery of the University Hospital Frankfurt, Germany, with subarachnoid haemorrhage and a small intracerebral haemorrhage from a ruptured left middle cerebral artery (MCA). Clinically she was graded as Hunt and Hess grade 3. Owing to the aneurysm morphology, endovascular coiling was not undertaken and the aneurysm was clipped 23 hours after the ictus. The initial postoperative period was uneventful until she became obtunded and developed a right hemiparesis on day 3. MRI with PWI and DWI was carried out. DWI showed no hyperintense signal changes (fig 1, panels A and B). Measurement of time to peak (TTP), mean transit time (MTT), relative regional cerebral blood volume ( $\mathrm{rCBV})$, and relative regional cerebral blood flow ( $\mathrm{rCBF}$ ) showed a severe perfusion deficit in the complete left MCA territory (fig lC). On the affected left side, rrCBF was reduced to 0.47 (frontal MCA territory) and to 0.32 (dorsal MCA territory) compared with the contralateral side. Relative regional $\mathrm{CBV}$ was 0.69 and 0.30 on the left side compared with the right in the frontal and dorsal MCA territories, respectively. Digital subtraction angiography was undertaken immediately after MRI and confirmed severe proximal focal vasospasm in the left $\mathrm{Cl}$ and $\mathrm{Ml}$ segments (fig 1D). Following TBA, which was done two hours after MRI, there was impressive widening of the $\mathrm{Cl}$ and $\mathrm{Ml}$ segments (fig 2D), and follow up MRI 70 hours after TBA showed resolution of PWI-DWI mismatch with exception of the terminal dorsal MCA territory (fig 2C). In this area there were hyperintense signal changes in DWI, indicating tissue infarction (fig 2B). In the anterior and middle MCA territory there were, however, no signs of infarction and PWI showed nearly complete normalisation of the initial perfusion deficit, with only 1.2 to 1.8 seconds delay compared with the contralateral regions of interest. Likewise the $\operatorname{rCBF}(\mathrm{rrCBV}$ ) increased to $0.80(0.84)$ in the left frontal but only to $0.39(0.40)$ in the terminal dorsal MCA territory.

During the remaining hospital stay the patient recovered and was discharged into a rehabilitation centre. At follow up examination six months after the ictus the patient was graded 2 on the modified Rankin scale.

\section{DISCUSSION}

In this case of severe vasospasm after subarachnoid haemorrhage, MRI identified a large area-that is, the complete left MCA territory-with a PWI-DWI mismatch. There was a delay of MTT of 6.7 to 16.4 seconds caused by vasospastic narrowing of the ipsilateral proximal $\mathrm{Ml}$ and $\mathrm{Cl}$

\footnotetext{
Abbreviations: DWI, diffusion weighted imaging; MCA, middle cerebral artery; MTT, mean transit time; PWI, perfusion weighted imaging; $r \mathrm{rCBF}$, relative regional cerebral blood flow; r rCBV, relative regional cerebral blood volume; $\mathrm{SAH}$, subarachnoid haemorrhage; TBA, transluminal balloon angioplasty; TCD, transcranial Doppler; TTP, time to peak
} 

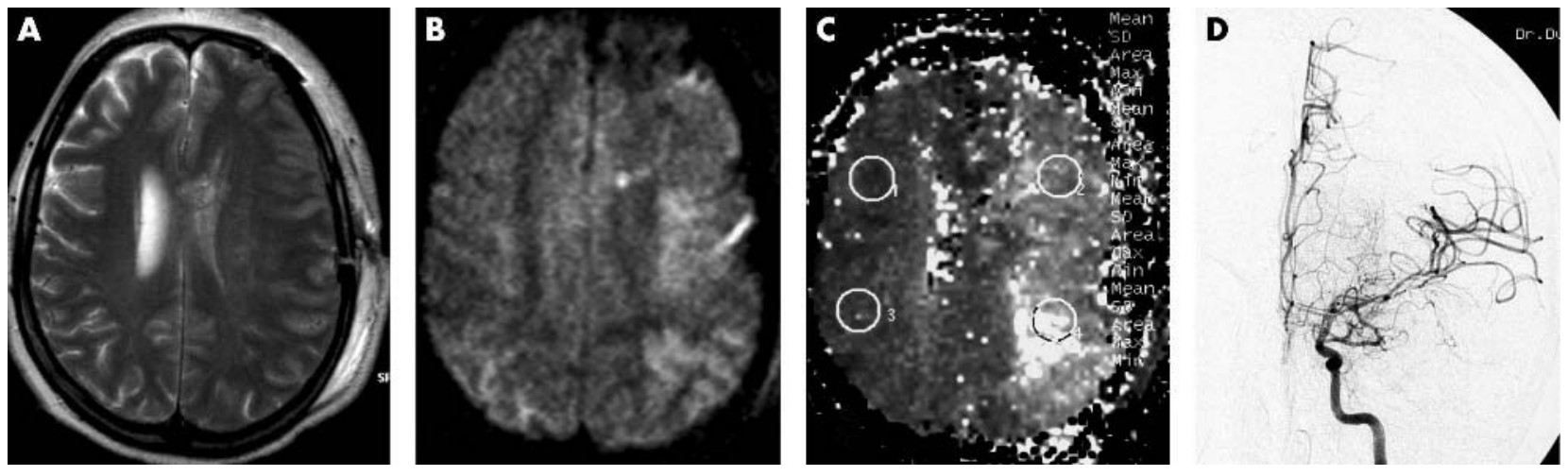

Figure 1 T2 (A) and diffusion weighted (B) images before transluminal balloon angioplasty showed no structural abnormality due to ischaemic infarction. However, on perfusion weighted imaging (C), the MTT (TTP) delay, as quantified between regions of interest (ROI) 1 and 2, was 6.7 seconds (5.9), and 16.4 seconds (16.1) between ROI 3 and 4. Digital subtraction angiography (D), carried out two hours later, revealed severe segmental vasospasm of the $\mathrm{Cl}$ and $\mathrm{Ml}$ segments.

segments, as detected by digital subtraction angiography. The stenoses could be successfully dilated by TBA, the patient recovered, and follow up MRI examination 70 hours after TBA showed that angioplasty not only had lasting haemodynamic effects but also prevented infarction of the complete MCA territory. Moreover the MCA territories with normalisation of the perfusion deficits did not undergo infarction, in contrast to the small area where the delay of MTT was reduced though still as long as 11.4 seconds compared with the contralateral side.

MRI including DWI and PWI sequences is very promising in subarachnoid haemorrhage to assess ischaemia and brain damage..$^{4-8}$ Review of the literature yielded one report of the use of PWI/DWI to select for angioplasty in severe vasospasm. The investigators assessed qualitatively a large perfusion deficit in the setting of a small lesion on DWI, and this area of oligaemia was reversed by angioplasty combined with a papaverine infusion, resulting in prevention of infarction and a good outcome. ${ }^{9}$ In the current study we additionally tried to quantify the perfusion deficit as well as the treatment effects of TBA.

The diagnosis and pathophysiology of cerebral ischaemia caused by vasospasm in subarachnoid haemorrhage is challenging. ${ }^{1}$ Angiographic measurement of vessel diameter detects anatomical narrowing of cerebral arteries in up to $70 \%$ which may be asymptomatic. ${ }^{10}$ Transcranial Doppler (TCD) is routinely used to detect vasospasm but has a very low predictive value, and even in patients who develop delayed neurological deficits TCD indices do not reflect cerebral perfusion. ${ }^{11}$ In our patient, TCD was not diagnostic for vasospasm and correlated with neither the clinical nor the radiological findings. Measurement of rCBF by a thermodilution probe is promising and yields absolute values of $\mathrm{CBF}$ but may miss the territory of misery perfusion. $^{12}$

The appeal of the tissue at risk concept in vasospasm is that it allows one to determine whether vasospasm is symptomatic - that is, that it leads to tissue ischaemia-as compared with other regions of interest. Furthermore, with this tool one can recognise whether the ischaemic tissue is still salvageable, potentially widening the window of opportunity for TBA. $^{13}$ Thus the decision to institute invasive and potentially harmful treatments such as TBA or triple-H therapy may be based on the results of MRI. ${ }^{14}{ }^{15}$ To reject angioplasty or triple- $\mathrm{H}$ therapy if tissue is already infarcted could reduce the side effects and complications by further improving the efficacy of these treatments. ${ }^{16-18}$ Once such measures are applied in a patient, the efficacy as well as the possible complications can be monitored by follow up MRI investigations.
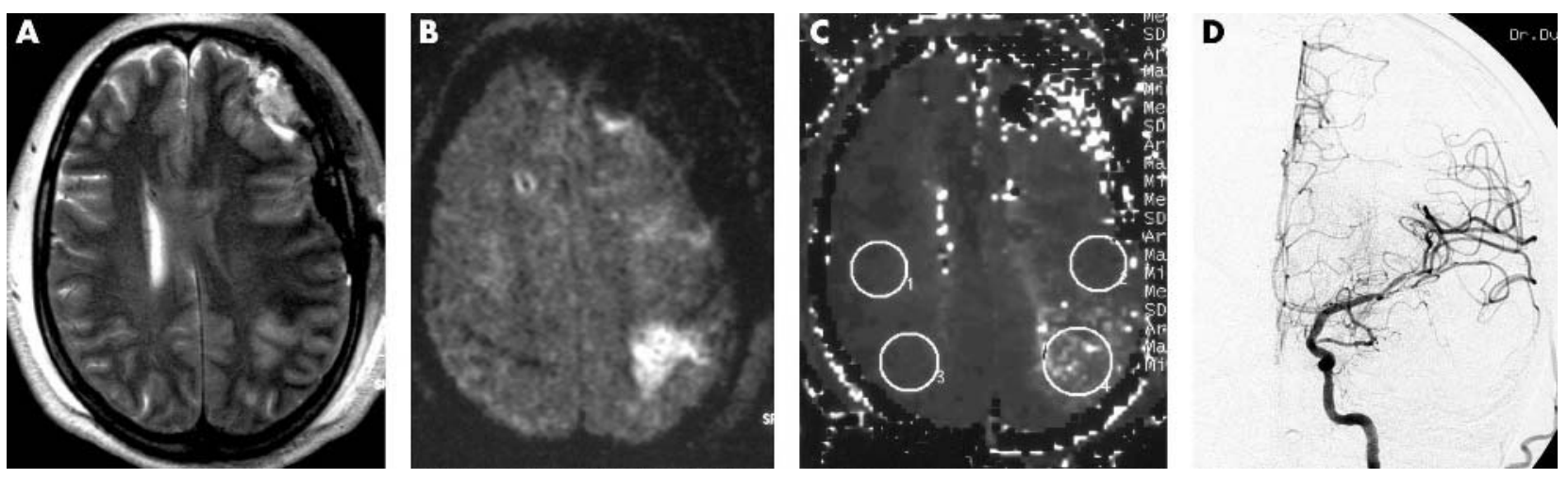

Figure 2 T2 (A) and diffusion weighted (B) images after transluminal balloon angioplasty show small hyperintense lesions due to infarction in the terminal dorsal middle cerebral artery (MCA) territory. Additional perfusion weighted imaging (C) 70 hours after dilatation of the left $\mathrm{Ml}$ and $\mathrm{Cl}$ segments (D) now showed normalisation of perfusion in the anterior and middle MCA territory with a reduction in the MTT (TTP) delay from 9.4 seconds (8.8) in the initial study to 1.8 seconds (1.2) between regions of interest (ROI) 1 and 2. The exception was an area in the terminal dorsal MCA territory which still had an MTT (TTP) delay of 11.4 seconds (10.8) as assessed between ROI 3 and 4 . This area is recognised as infarcted tissue on diffusion weighted imaging (B). 
In ischaemic stroke the tissue at risk concept is well established and PWI/DWI studies are part of the clinical routine before systemic or intra-arterial lysis. ${ }^{3}$ In acute ischaemic stroke a perfusion deficit of more than six seconds in the mismatch region is followed by expansion of tissue infarction if it is not rapidly reversed. ${ }^{19}{ }^{20}$ Even a less severe perfusion deficit of 2.0 to 2.5 seconds may represent dysfunctional and potentially salvageable tissue rather than oligaemia with no risk for infarction. ${ }^{21}$ These thresholds may not apply to the scenario in vasospasm after subarachnoid haemorrhage. In contrast to embolic or thrombotic ischaemic stroke, the development of misery perfusion is presumably not a sudden event but rather evolves over hours or days. On the one hand this time allows for some collateralisation to develop; on the other hand a less severe reduction in perfusion, if of long enough duration, can also lead to infarction. ${ }^{22}$ In the current case of severe vasospasm we do not know for certain whether that part of the MCA territory with deficits of 6.7 and 9.4 seconds would have been infarcted without TBA or not. Although it is very likely that infarction would have occurred without TBA more research will have to be done to clarify the thresholds for tissue infarction in vasospasm and the sensitivity and specificity of PWI/DWI. The best time for undertaking a PWI/DWI investigation after subarachnoid haemorrhage, and whether the method can detect a mismatch in clinically asymptomatic patients, remain to be determined. A drawback of all relative perfusion measurements in different regions of interest is that global changes - that is, changes in both hemispheresmay not be detected. ${ }^{23}$

\section{Conclusions}

PWI/DWI imaging could be used on a routine basis to monitor patients with subarachnoid haemorrhage for tissue at risk, even in sedated patients where clinical surveillance is not possible. We believe that the tissue at risk concept is a promising tool in the detection of cerebral vasospasm as well as in monitoring the effects of TBA. However, the concept requires further development, especially in patients with higher grading on the Hunt and Hess scale.

\section{Authors' affiliations \\ J Beck, A Raabe, V Seifert, Department of Neurosurgery, Johann Wolfgang Goethe-University, Frankfurt am Main, Germany H Lanfermann, S Weidauer, Institute of Neuroradiology, Johann Wolfgang Goethe-University \\ Competing interests: none declared}

Correspondence to: Dr Jürgen Beck, Department of Neurosurgery, Johann Wolfgang Goethe-University Frankfurt am Main, Schleusenweg 2-16, 60528 Frankfurt am Main, Germany; J.Beck@em.uni-frankfurt.de

Received 16 January 2004

In revised form 25 March 2004

Accepted 31 March 2004

\section{REFERENCES}

1 Minhas PS, Menon DK, Smielewski P, et al. Positron emission tomographic cerebral perfusion disturbances and transcranial Doppler findings among patients with neurological deterioration after subarachnoid hemorrhage. Neurosurgery 2003;52:1017-22.

2 Moseley ME, de Crespigny AJ, Roberts TP, et al. Early detection of regional cerebral ischemia using high-speed MRI. Stroke 1993;24:160-5.

3 Schellinger PD, Fiebach JB, Hacke W. Imaging-based decision making in thrombolytic therapy for ischemic stroke: present status. Stroke 2003;34:575-83.

4 Condefte-Auliac S, Bracard S, Anxionnat R, et al. Vasospasm after subarachnoid hemorrhage: interest in diffusion-weighted MR imaging. Stroke 2001;32:1818-24.

5 Leclerc X, Fichten A, Gauvrit JY, et al. Symptomatic vasospasm after subarachnoid haemorrhage: assessment of brain damage by diffusion and perfusion-weighted MRI and single-photon emission computed tomography Neuroradiology 2002;44:610-16.

6 Phan TG, Huston J, Campeau NG, et al. Value of diffusion-weighted imaging in patients with a nonlocalizing examination and vasospasm from subarachnoid hemorrhage. Cerebrovasc Dis 2003;15:177-81

7 Griffiths PD, Wilkinson ID, Mitchell P, et al. Multimodality MR imaging depiction of hemodynamic changes and cerebral ischemia in subarachnoid hemorrhage. Am J Neuroradiol 2001;22:1690-7.

8 Rordorf G, Koroshetz WJ, Copen WA, et al. Diffusion- and perfusionweighted imaging in vasospasm after subarachnoid hemorrhage. Stroke 1999;30:599-605.

9 Bracard S, Anxionnat R, Auliac S, et al. Relevance of diffusion and perfusion weighted MRI for endovascular treatment of vasospasm in subarachnoid hemorrhage. J Neuroradiol 2001;28:27-32.

10 Heros RC, Zervas NT. Subarachnoid hemorrhage. Annu Rev Med 1983;34:367-75.

11 Vora YY, Suarez-Almazor M, Steinke DE, et al. Role of transcranial Doppler monitoring in the diagnosis of cerebral vasospasm after subarachnoid hemorrhage. Neurosurgery 1999;44:1237-47.

12 Vajkoczy P, Horn P, Thome C, et al. Regional cerebral blood flow monitoring in the diagnosis of delayed ischemia following aneurysmal subarachnoid hemorrhage. J Neurosurg 2003;98:1227-34.

13 Rosenwasser RH, Armonda RA, Thomas JE, et al. Therapeutic modalities for the management of cerebral vasospasm: timing of endovascular options. Neurosurgery 1999:44:975-9.

14 Elliott JP, Newell DW, Lam DJ, et al. Comparison of balloon angioplasty and papaverine infusion for the treatment of vasospasm following aneurysmal subarachnoid hemorrhage. J Neurosurg 1998;88:277-84.

15 Muizelaar JP, Zwienenberg M, Rudisill NA, et al. The prophylactic use of transluminal balloon angioplasty in patients with Fisher grade 3 subarachnoid hemorrhage: a pilot study. J Neurosurg 1999;91:51-8.

16 Firlik AD, Kaufmann AM, Jungreis CA, et al. Effect of transluminal angioplasty on cerebral blood flow in the management of symptomatic vasospasm following aneurysmal subarachnoid hemorrhage. J Neurosurg 1997;86:830-9.

17 Polin RS, Coenen VA, Hansen CA, et al. Efficacy of transluminal angioplasty for the management of symptomatic cerebral vasospasm following aneurysmal subarachnoid hemorrhage. J Neurosurg 2000:92:284-90.

18 Andaluz N, Tomsick TA, Tew JM, et al. Indications for endovascular therapy for refractory vasospasm after aneurysmal subarachnoid hemorrhage: experience at the University of Cincinnati. Surg Neurol 2002;58:131-8.

19 Thiij VN, Adami A, Neumann-Haefelin T, et al. Relationship between severity of MR perfusion deficit and DWI lesion evolution. Neurology 2001;57:1205-11.

20 Neumann-Haefelin T, Wittsack HJ, Wenserski F, et al. Diffusion- and perfusion-weighted MRI. The DWI/PWI mismatch region in acute stroke. Stroke 1999:30:1591-7

21 Hillis AE, Wityk RJ, Tuffiash E, et al. Hypoperfusion of Wernicke's area predicts severity of semantic deficit in acute stroke. Ann Neurol 2001;50:561-6.

22 Hossmann KA. Viability thresholds and the penumbra of focal ischemia. Ann Neurol 1994:36:557-65.

23 Neumann-Haefelin T, Wittsack HJ, Fink GR, et al. Diffusion- and perfusionweighted MRI: influence of severe carotid artery stenosis on the DWI/PWI mismatch in acute stroke. Stroke 2000;31:1311-17. 